\title{
Applying the Modified Early Warning Score (MEWS) to assess geriatric patients in home care settings: A qualitative study of nurses' and general practitioners' experiences
}

Kristin Jeppestøl ( $\nabla$ kristin.jeppestol@tvedestrand.kommune.no)

Tvedestrand kommune https://orcid.org/0000-0002-1932-8685

Line Kildal Bragstad

Universitetet i Oslo

Marit Kirkevold Kirkevold

Universitetet i Oslo

\section{Research article}

Keywords: Early warning score system, home care, elderly, geriatric, acute functional decline, clinical decision-making, community care

Posted Date: October 31st, 2019

DOI: https://doi.org/10.21203/rs.2.16666/v1

License: (c) (i) This work is licensed under a Creative Commons Attribution 4.0 International License. Read Full License 


\section{Abstract}

Background : Acute functional decline is a common clinical syndrome in geriatric health care and is typically characterised by nonspecific symptoms and presentations with a mix of physical, psychological, social and functional manifestations. Early warning score (EWS) systems are widely implemented in nursing homes and home care to detect clinical deterioration. The effects of EWS systems have been thoroughly evaluated in hospital care settings, but few studies have evaluated EWS systems in community healthcare. The purpose of this study was to describe the experiences of registered nurses and general practitioners experiences when using the Modified Early Warning Score (MEWS) to support clinical reasoning and decisionmaking with geriatric home care patients who suffer from acute functional decline.

Method: A qualitative methodology was used with a descriptive exploratory design. Data were collected from seven focus group interviews. General practitioners (GPs), and registered nurses (RNs) were purposively sampled from large, medium and small municipalities in Norway. Data were analysed using an inductive content analysis method.

Results: MEWS was used as an additional decision-making tool with elderly home care patients when acute functional decline was detected. RNs and GPs highlighted that MEWS supported the clinical reasoning and decision-making process. Additionally , comprehensive reasoning skills and specific knowledge of the patients were needed. RNs identified the need for contextual adjustments to the use of MEWS in home care settings. Implementing MEWS has improved the collaboration and clinical practice of RNs and GPs. The adherence to MEWS follow-up recommendations was adjusted to the home care setting, accounting for potentially limited medical availability.

Conclusion : MEWS supported RNs and GPs in conducting comprehensive clinical assessments and reasoning when acute functional decline was detected. Interdisciplinary communication and collaboration appeared to be strengthened, and the GP's work was streamlined. Several limitations were identified with the use of MEWS reference values with geriatric patients, which could lead to ambiguity and misjudgements. MEWS trigger recommendations were experienced as inappropriate to comply within home care. This study identifies the need for a modified evidence-based EWS adjusted for geriatric patients in home care.

\section{Introduction}

The transfer of responsibility for medical treatment from the specialist to primary health care leads the community health care services to provide advanced health care services to the elderly population [1]. Proactive measures developed for hospital practice, such as early warning score systems (EWS), are widely implemented in nursing homes and home care in order to detect clinical deterioration and start medical treatment at an early stage at the lowest level of care [2]. Despite the lack of research into the effect of these systems on elderly persons in general and in the community health care service in particular, the Norwegian patient safety programme recommends that EWS be applied [3] in all services.

\section{Background}

\subsection{Acute functional decline}

The elderly population receiving home care are a frail group of individuals with complex diseases and needs for comprehensive health care [4-6]. When frail elderly persons become acutely ill, the symptoms are often nonspecific, with an atypical presentation in a mix of physical, psychological, social and functional manifestations [7-11]. In Norway and in this paper, this group of symptoms is referred to as 'acute functional decline'. Internationally, the term 'geriatric syndrome' is often used to describe the same condition, and there is a lack of consensus on the definition of the condition [8, 9]. In this paper, the following symptoms characterise acute functional decline: physical symptoms, such as fatigue, weakness, loss of Activities of daily living (ADL) capacity, loss of appetite, falls, incontinence and psychological symptoms, such as loss of attention, initiative and motivation and general cognitive impairment. Furthermore, acute functional decline is often caused by somatic and common diseases in the elderly $[7,11]$, such as infections and cardiovascular, cerebrovascular, pulmonary, neurological, musculoskeletal, metabolic and endocrine diseases. Medication side effects and trauma can also cause acute functional decline [7] and hospital admission is often required. An interdisciplinary and comprehensive geriatric approach is recommended to improve patient 
outcomes [12]. The magnitude of the symptoms and functional manifestations may be interpreted by family members or health care professionals as a trigger for the need of a higher level of care rather than signs of acute disease [7]. However, identifying the symptoms and targeting the causes of acute functional decline and treatment of the cause is crucial to prevent frailty $[7,12$, 13].

\subsection{Fundamental aspects of practice}

Health care professionals need broad clinical expertise to identify acute illness and perform systematic follow-up to collaborate with GPs to address the cause of the functional decline and provide proper health care in time [4]. Clinical reasoning and decision-making are fundamental aspects of home care, central to independent practice, and are the foundation for health professionals to perform the right measures in different situations and contexts. Higgs and Jones [14] describe clinical reasoning and decision-making as the sum of thinking and decision-making processes in clinical practice. Furthermore, Higgs and Jones (2008) have recognised clinical reasoning and decision-making as a complex and multidimensional phenomenon that includes individual cognitive and non-cognitive processes in interdisciplinary teamwork [14].

In the Norwegian care sector, health personnel with college education (RNs) represent $35 \%$ of the employees, and health personnel with upper secondary education (nurses' assistants) represent $40 \%$. The remaining $25 \%$ employees are represented by unskilled workers [15].

Organising for the proper competence and continuity in home care is important to improve patient safety but is, at the same time challenging, due to a serious RN shortage [16]. Although continuity of health care is a legislated, political and health services goal [17], a study of continuity in Norwegian home care shows that older recipients of daily and long-term home care must cope with deal with many different people when they receive health care in their own home [18]. This lack of interpersonal continuity, may threaten the ability of the personnel to recognise subtle changes in the health conditions of frail older persons.

\subsection{Formal home care service}

In Norway, the municipalities hold the responsibility of primary health care for the elderly living at home. Elderly individuals in need of assistance with personal and instrumental activities of daily living receive individual help provided by home care services according to their needs. The service hours are set for a certain number of visits and times for daily or around-the-clock care; service is based on individual assessments and is adjusted as needed [19]. In the home care team, registered nurses (RNs) have the highest formal competence and education, and they hold the responsibility for providing care and assessing acutely ill patients in cooperation with nurses' assistants and unskilled workers. The overall responsibility of medical treatment is assigned to the patient's general practitioner (GP), who cooperates with home care nurses mainly through communication by telephone or electronic messages. All GPs are required to perform out-of-hours emergency service duty. The out-of-hours emergency service ensures the medical availability in evenings, nights, weekends and public holidays. An intermunicipal out-of-hours emergency service can be contacted when the GP's office is closed if there is a need for acute medical supervision in home care.

\subsection{Early warning score systems in hospital and pre-hospital settings}

EWS, or physiological 'track-and-trigger' systems, were designed to support health care professionals in identifying and responding to acutely unwell patients at risk of clinical deterioration [20]. The effects of EWS systems have been thoroughly evaluated in hospital care settings. Various scoring systems used in hospital settings perform well for predicting cardiac arrest and death within 48 hours [21-23]. Furthermore, studies show that EWS cannot replace clinical judgement, and EWS in combination with clinical judgement is important for making clinical decisions in pre-hospital- and hospital settings [20, 24-28]. Several studies have noted challenges when using EWS in a variety of clinical settings and patient groups, and point out a need to adjust EWS to assure proper use [20, 23-25]. 
A study showed that EWS and Rapid Response Systems (RSS) improved RNs' decisions about whether to summon help as well as their collaboration and communication during the referral process [26]. Conversely, RNs found it challenging to contact the physician when they were concerned about the patient, based on their clinical intuition, and when the score was low. The findings from that study could indicate that the hierarchical hospital culture might influence the nurses' ability to assess and manage patients in clinical deterioration [26].

Studies investigating how RNs used an EWS tool in acute hospital settings revealed that nurses' clinical judgement was challenged regarding escalation protocols, especially when parameter adjustments were not performed by senior medical personnel for patients with vital parameters outside the reference values due to chronic diseases [24, 25]. RNs working in a hospital setting also reported challenges with getting the physicians to review the patient according to the escalation protocol $[24,25]$.

Studies indicate that EWS in pre-hospital settings could identify patients at risk of deterioration and in need of hospitalisation; patients at low risk of deterioration could be safely managed at home [29].

One study explored RNs' and GPs' experiences of using the track-and-trigger system National Early Warning Score (NEWS) in prehospital, primary and community health care settings [20]. The participants reported that NEWS could support clinical decisionmaking around the escalation of care, and could provide a clear means of clinical communication between clinicians and across different healthcare organisations. However, modifications were needed to make the tool relevant for some patient populations in community services [20].

\subsection{The EWS of southern Norway}

In the Hospital of Southern Norway, an EWS system for early identification of life-threatening conditions (Norwegian acronym: TILT) was developed in 2014 [30]. TILT consists of a five-item Modified Early Warning Score (MEWS), an observational curve for vital parameter registration, and an e-learning system for training healthcare staff. This study will focus on the EWS tool MEWS. MEWS consists of five items of vital parameters: heart rate, temperature, respiratory rate, blood pressure and level of consciousness (CNS) $(14,15)$. The vital signs are measured, and a score is calculated based on the vital measurements. These parameters provide information about the person's physical condition and vital parameters that deviate from reference values, represent scores in points and colour codes and generate support concerning when the measurements are to be repeated and when to contact a physician. A score of 0 triggers new measurements every 24 hours; a score of 1 triggers new measurements in 8-12 hours; a score of 2 triggers new measurements every 4-8 hours; a score of 3-4 triggers new measurements every 1-4 hours; scores of 3 or 4 indicate contacting a physician. A MEWS score of 4 or higher requires healthcare personnel to contact a physician. In the MEWS version used in the Hospital of Southern Norway and the municipalities in this study (TILT), oxygen saturation was also a part of the assessment but did not contribute to the score. A sudden drop of saturation (< $90 \%)$ requires the health staff to contact a physician as well as a general concern for the person's condition [30] (Figure 1).

There is a lack of studies that have evaluated track-and-trigger systems, such as MEWS, in community health care. However, many Norwegian municipalities have implemented MEWS and other EWS systems in home care and nursing homes in recent years, encouraged by the Norwegian Patient Safety Program [3]. Knowledge is needed on how these scoring systems impact the care provided by RNs and GPs in community care.

The overall purpose of this study was to describe RNs' and GPs' experiences with the EWS tool MEWS to support clinical reasoning and decision-making in work with the elderly who receive home care and suffer from acute functional decline.

The two research questions were as follows:

How is the early warning score tool MEWS used by RNs and GPs in the home care service?

Which strengths and limitations are identified by RNs and GPs related to the use of MEWS as support in clinical reasoning and decision-making in home care? 


\section{Method}

\section{1 Design, setting and recruitment}

This is a qualitative interview study with a descriptive exploratory research design [31]. Semi structured focus group interviews were conducted to explore issues and challenges regarding the use of MEWS in clinical practice from the participants' subjective perspectives [32, 33].

The administrative leaders of the municipalities approved the RNs to participate in the interviews and appointed a contact person in the home care team who recruited the RNs. The RNs were chosen through purposive sampling to ensure that they had experience with MEWS in home care. The first author contacted the district medical officers of the municipalities for approval and contact information for current GPs who had experience with using MEWS and cooperating with home care. Five GPs were recruited by a medical officer, and other department managers in the health care service and three physicians were recruited by the first author via email and telephone.

\subsection{Data collection}

Data collection started in September 2018 and was completed in November 2018. Participants were recruited from one large (greater than 20,000 inhabitants), three medium (less than 20.000 inhabitants), and three small (less than 5000 inhabitants) municipalities in southern Norway [34]. These specific municipalities were included in the study with the reason that all municipalities have collaborated in training and implementation of the same EWS.

Seven focus group interviews were conducted in the participant's workplace and lasted an average of 68 minutes (range 53-78 minutes). An interview guide with topics and questions related to the advantages and challenges of the use of MEWS in clinical practice was used in all interviews. All interviews were audiotaped to ensure accuracy for transcription and analysis. All authors participated in the data collection. MK moderated the two first interviews and $\mathrm{KJ}$ attended as co-moderator. $\mathrm{KJ}$ moderated the five remaining interviews, and LKB attended as co-moderator.

\subsection{Ethical considerations}

This study was approved by The Norwegian Regional Committees for Medical and Health Research Ethics (2018/469). All the participants signed the consent form, assuring voluntary participation and confidentiality. All data are securely stored in the University of Oslo's Services for Sensitive Data (TSD).

\subsection{Data analysis}

All interviews were transcribed verbatim with the transcription software f4transkript [35]. All transcripts were imported into the qualitative analysis software Nvivo12 [36]. The data were analysed by qualitative thematic content analysis, inspired by Green and Thorogood [37] and Tjora [38].

\subsubsection{Coding and categorisation}

$\mathrm{KJ}$ and LKB coded the first interview together to identify codes and ensure consensus. Furthermore, the second interview was coded by KJ and LKB separately. New and similar codes were discussed, and the degree of agreement was assessed in the software Nvivo12 and resulted in 95-98\% agreement. KJ coded the remaining five interviews. The first coding process resulted in a total of 114 codes. All interviews were read again, and codes were grouped into similar content for 24 categories. The categories were discussed before the subsequent analysis was commenced. In the ensuing analysis, nine categories were found to be relevant to the research questions of this study. Furthermore, two main themes emerged in the process of analysis. The first 
theme was "MEWS as an additional clinical decision-making-tool" and the second main theme was "Adjusting the use of MEWS to non-institutional care setting". Several codes were included in these main categories; examples are presented in Table 2.

\section{Results}

\section{Participants}

Altogether, 44 healthcare personnel were included in this study, 36 RNs, and eight GPs. All RNs participated in direct patient care. GPs from each municipality participated in all interviews, except for one municipality where only RNs were present at the interview. All physicians worked as GPs. Four GPs had additional functions in the municipal health service, such as supervisory doctors in nursing homes and municipal superiors. Characteristics of the participants are presented in Table 1.

\subsection{MEWS as an additional clinical decision-making-tool}

MEWS was an important tool for RNs and GPs in facilitating clinical reasoning and decision-making when acute functional decline was detected. However, several challenges were identified related to the use of MEWS with elderly care recipients in home care.

\subsubsection{Vague and diffuse symptoms indicated the use of MEWS}

RNs described that MEWS was used when newly emerging functional decline and vague, diffuse symptoms deviating from the patient's habitual condition and daily function was detected:

'He was quite lucid, but his gait function was reduced. That was kind of how you noticed it [the functional decline]' (RN-4).

The detection of symptoms and functional decline could be detected and reported by RNs or other health care staff, the patient's relatives or the patients themselves:

'I guess it's like... when you see the care recipient, and he doesn't behave like he normally does. Either you see that he is clammy or sweaty or that he says: "I'm not....there's something today... I don't know what it is, but I'm not like I usually am". Then we tend to do $M E W S^{\prime}(R N-3)$.

RNs highlighted the importance of listening to what the patients were telling them about their chief complaint in combination with obtaining the medical history while trying to identify the cause of the functional decline. Minor deviations in vital parameters could be detected in these cases: for example, hypo- or hypertension, or tachycardia or bradycardia, which could be reported to the GP for adjustment of medication.

Furthermore, more severe deviations, such as dehydration, infections and sepsis, could also be detected. RNs in all municipalities gave examples, describing that MEWS had helped them detect sepsis. RNs explained that sepsis rapidly develops in elderly patients, and found it difficult to recognise without using objective measurements:

'I have experienced it twice at work, where the patients in their habitual state could complain about symptoms like "feeling weak and unwell." And MEWS showed that both had very low blood pressure! And to be honest, I don't think we would have detected it otherwise.'(RN-18).

\subsubsection{MEWS in home care requires comprehensive clinical reasoning}

RNs and GPs emphasised that geriatric patients- had many chronic diseases, which often caused them to have several diffuse and vague symptoms and deviating vital parameters in their habitual state. RNs and GPs emphasised that the patients' habitual state must be taken into account during the assessment when performing the MEWS measurements. Preferably, the first time 
MEWS was performed for a patient in home care, and it was done to document the patient's habitual MEWS scores and used as a baseline measurement to compare subsequent MEWS scores.

RNs expressed several challenges in interpreting the reference values as well as the trigger recommendations MEWS proposes to support clinical reasoning and decision-making:

'We work a lot with geriatric patients, and I think that MEWS is not adapted to geriatric patients. It's kind of like it should be suitable for me if I'm hospitalised'. (RN-4)

Both GPs and RNs in all participating municipalities found that the reference values of the respiratory rate, in particular, were too sensitive:

'I think that the respiratory rate score is very low; it's for a healthy person, and we really have none of those [in our care]' (RN-2).

MEWS reference values of normal respiratory rate could, according to RNs, lead to lack of responsivity, and some feared that they would not react and detect early signs of clinical deterioration because they had experienced that the majority of elderly patients had a respiratory rate above 14 , which, according to MEWS, was considered to be abnormal:

'We are very often asked to assess MEWS scores performed by skilled workers, and let's say they report a score 1 because of elevated respiratory rate.... Well, I sometimes fear that I overlook a MEWS score that I really should react to' (RN-4).

The reference values for blood pressure were also mentioned as too wide as were the reference values for temperature, which in elderly persons can be falsely low, due to medication use. MEWS normal reference values for temperature, may camouflage fever as well as a late physiological response in geriatric patients.

RNs sometimes avoided performing MEWS when they reasoned that the clinical situation was normal because it would create additional work with the assessment and follow-up since most patients would have parameters that resulted in a MEWS score of 1 or 2 , which would trigger a type of follow-up.

RNs and GPs emphasised that the MEWS score was only an additional tool and that the importance of knowing the patients' normal function and vital parameters in their habitual condition played an important role to support the clinical reasoning and decision-making process.

\subsubsection{The importance of knowing the care recipient}

The RNs explained that knowing the patient was important to understand the clinical situations and make proper decisions, and knowing the patient emerged as a common theme in all interviews with both RNs and GPs. Knowing the patient included their detailed knowledge about how they managed daily functional activities, knowing their diagnosis and vital parameters in their habitual state. The GPs also expressed the importance of the RNs' knowledge of the patient's habitual state when they assessed MEWS scores and symptoms of acute functional decline, and that this relationship strengthened communication and cooperation regarding decision-making about whether to start or wait with medical treatment or if the patient should be admitted to the hospital.

RNs also highlighted the importance of providing 'additional information' when acute functional decline occurred, and they attempted to find out what caused the functional decline by performing additional examinations, like CRP tests and urine samples.

'You have to consider what's causing it [the functional decline] if there is a change in a MEWS score' (RN-33).

All home care teams in the municipalities are intended to have habitual vital parameters documented in all home care patients' electronic records. Some home care teams routinely conferred with the GP when they had performed MEWS baseline measures to ensure that vital signs, which were often outside the cut off scores, were considered normal for the individual. Conferring with the GP was particularly the case for patients with congestive heart failure, COPD and other chronic disorders that caused 


\subsection{Adjusting to the use of MEWS in a non-institutional care setting}

MEWS's trigger recommendations were described by RNs as challenging in the context of home care. Unlike the hospital setting, home care nurses do not see the patients $24 / 7$, and they did not have immediate access to medical services in case of acute illness. RNs and GPs highlighted that the use of MEWS impacted the quality of communication and cooperation, although adjustments to the use of MEWS related to medical availability as well as to the patients, was required.

\subsubsection{Implementation of MEWS support interdisciplinary cooperation in clinical reasoning and decision-making}

The GPs expressed that they received more specific referrals and more complete and objective information from the RNs along with the clinical assessment and other examinations, such as CRP and urine samples, after implementing MEWS:

'A lot of the work is already done when we communicate with the nurses now. Before MEWS, there were a lot of telephone calls and pulses were not taken, nor the respiratory rate... so this has eased our job when we assess by telephone. It has especially eased my workday'(GP-36).

According to GPs, MEWS has led the RNs to an increased awareness related to performing vital measurements and assessment of both objective and subjective data:

'Really, before MEWS... I could ask the nurse... what is the respiratory rate? It really didn't happen. It didn't happen before...or...I didn't get the answer to these questions. It's something that has happened after implementing MEWS. I don't think the nurses counted the respiratory rate. They would say shortness of breath or not shortness of breath or the patient is breathing fast'(GP23).

The RNs also reported that their observational skills had improved and they now assessed all parameters in a more comprehensive way than before. Several GPs reported that the implementation of MEWS had improved their ability to conduct proper medical reasoning and decisions for the patients, which led to a general improvement of clinical practice:

'We were often presented snapshots from a situation before MEWS. But now, we get more repeated observations. And that makes it really useful to assess the clinical condition, and it's much easier to assess together with the nurses and decide which measures to initiate'(GP-36).

'I think it is easier now [after the introduction of MEWS] to make assessments early in the process. It has changed the course of treatment for the patients. We can initiate medical help early, or we can say no, it's safe to wait. You might avoid unnecessary hospital admissions, for example' (GP-14).

\subsubsection{Adjusting MEWS to the context of home care}

RNs expressed that it was not always feasible to follow MEWS' trigger recommendations in home care because of geographical distances and work lists with limited flexibility:

'To achieve continuity all the time... it's fine if they live close to the base, but if the patient lives far away, for example, a 40 minute drive, and you are supposed to perform a MEWS again two hours later [it becomes unfeasible]' (RN-2).

RNs made decisions regarding how and when to follow up after taking a MEWS measurement based on their own clinical reasoning, often consulting other colleagues, and if possible, consult with other health professionals for interdisciplinary assessment: 
'It should be said that we are not that structured when it comes to the follow-up intervals. But sure, we do our own reasoning, even if it's score 3 or 2'(RN-44).

However, some RNs expressed, that the colour codes organised the assessments and that follow-up intervals were systematised; colour codes visualised the severity of the patients' health condition and provided concrete guidelines for follow-up.

'But what I think is ok with the colour codes in the MEWS card is that it tells me, if it's a yellow score, the patient should be followed up in a certain period of time. If it's a score 4, we are contacting a physician. We can sometimes doubt if we should contact the physician, but when it's a score 4 , we do contact the physician'(RN-26).

A MEWS score of 0 was easy to assess, according to RNs and GPs, because they considered the clinical situation as stable and normal, but were aware of the possibility to make incorrect decisions if they relied solely on objective parameters:

'There is a risk of blindly trusting the measurements and not interpreting the clinical situation. [MEWS] is a starting point, but there can be serious misjudgements'. (RN-44)

A MEWS score of 1-3 resulted in a dilemma for further clinical reasoning and decision-making, especially if the healthcare provider did not know the patient nor had access to habitual vital parameters in the electronic patient records. A MEWS score higher than 3, and if clinical indications for acute illness were present, were situations that RNs and GPs found easy to assess because they reasoned the situation as severe. If RNs considered the situation to be unstable and new MEWS measurements had to be done beyond the planned work, priority was given to new measurements, and they organised to do the follow-ups. They also considered other factors, such as whether the patient lived alone, the distance from the home care base and if it was close to a weekend when the GP office was likely to be closed for several days in the reasoning and decision-making process. If the MEWS score or the patient's clinical condition indicated frequent measurements because of an unstable health condition, or if the patient had deteriorated significantly, the RNs often reasoned that it was not prudent to stay at home.

These situations often led RNs to contact the GP or the out-of-hours emergency service, and the patient was often admitted to a higher level of care, such as a nursing home, the municipal emergency care unit or the hospital.

\subsubsection{Limited medical availability in home care required a plan B}

RNs described the municipal medical service as limited, and the GPs expressed that inquiries from RNs in home care had to be done between seeing the planned patients or after working hours. RNs reported that much time during the workday was used to contact and communicate with the GP, who was present during office hours on weekdays:

'I see that it matters what weekday it is [when MEWS is used]. If it is Friday and you know in a way that the patient [may need medical attention] Saturday or Sunday, then you have to think differently. If it is 14 hours until Monday and the medical office opens again...there are many things to consider' $(R N-20)$.

RNs found GPs' knowledge of the patients important and facilitated the GP-made decisions about further treatment and followup in collaboration. The specific knowledge was related to the fact that the GPs often had followed their medical condition for many years. Dilemmas would occur for RNs if the GPs were not available, if they worked part time or had other duties in the municipality. RNs expressed that they often reasoned the patient's health condition too complex - and they found it unsafe-for an intern or another GP in the out-of-hours emergency service to make the right clinical decisions:

"We think that we do not want to bother the patients or an intern at the out-of-hours emergency service by interfering with a patient that we reason to be in risk of deterioration on a Sunday if the care recipient has a well-functioning relationship with his or her general practitioner. In these cases, we'll wait until Monday'(RN-20).

The limited medical availability led RNs to try constantly to be ahead in terms of planning and foreseeing tentative scenarios, especially potential scenarios that could happen during evenings, weekends or holidays when the GPs were not available: 
'We especially plan for the holidays. If we see a patient is ill, then we think "OK, we must have a plan A. If plan A is not working, then we have to have a plan B". Then we might avoid contacting out-of-hours emergency service and hospital admission'" (RN44).

\section{Discussion}

This study describes RNs' and GPs' experiences with MEWS in the community setting. In the following, we discuss identified strengths and limitations related to the use of MEWS as support in clinical reasoning and decision-making in home care.

\subsection{Implementing MEWS has improved practice}

This study shows that RNs and GPs thought MEWS was successfully implemented as an important shared interdisciplinary tool to assure comprehensive clinical assessment and communication of vital parameters in the community care setting.

Furthermore, MEWS helped RNs in home care to perform and systemise the objective information of the patient, which could support their clinical reasoning and decision-making when diffuse and vague symptoms occurred in elderly patients.

According to the participants in our study, MEWS has strengthened the collaboration and communication between health care personnel in general, and between RNs and GPs in particular. The present study supports findings from earlier studies from other settings, $[20,23,26]$ where the use of EWS systems to communicate objective findings improved communication between health professionals.

Studies performed in hospitals showed that RNs found it challenging to contact the physician when they were concerned about a patient, based on their clinical intuition, but the score was low [26]. In contrast to studies conducted in the hospital setting, no impeding hierarchical structure in communication between RNs and GPs was identified in our study. Furthermore, RNs found that their inquiries were taken seriously and GPs expressed that they trusted the RNs' expertise in home care. GPs expressed the importance of integrating RNs' clinical knowledge and their concern regarding the patient's state of health as an important part of the reasoning and decision-making process, even if the vital parameters were normal. GPs received a complete set of objective vital parameters from RNs. These data, including the results of additional examinations and the subjective health history, improved the GPs' ability to work efficiently and make proper medical decisions for the patients. The present study supports findings from studies performed in other pre-hospital settings, that RNs experienced, that identification of potentially life-threatening conditions for elderly patients, such as infections and sepsis, were identified and treated earlier [29, 39] with EWS.

The results of this study indicate that the implementation of MEWS contributed to improved practice. However, comprehensive challenges with parameters and reference values in MEWS with geriatric patients were identified.

The present study supports/corroborates findings from earlier studies from other contexts/settings that MEWS

\subsection{Challenges in applying MEWS with geriatric home care patients}

Studies have highlighted challenges with using EWS systems in a variety of patient groups and point out the need to adjust EWS to ensure its proper use [20,23-25]. In the present study, RNs and GPs experienced that elderly patients frequently have vital values outside the MEWS reference values in their habitual state. All parameters and reference values in MEWS were discussed by RNs and GPs, and they agreed that the values were not adjusted for the majority of elderly patients in home care.

Normal respiratory rate values were found to be too sensitive for elderly patients in general, and COPD and heart failure patients in particular. Normal values of systolic blood pressure were experienced as too broad, and MEWS did not trigger measures with significant drops or rises from the patients' habitual values. Normal temperature values were too wide and not sensitive enough to track infections early. Oxygen saturation did not generate scores in MEWS and did not take into account patients suffering from congestive heart failure or COPD. Newly emerged confusion did not generate points in determining the level of consciousness, which is a significant symptom and factor in raising concern for acute illness in elderly patients [7]. However, 
there are several EWS systems with reference values that could better support the RNs' challenges when using MEWS with geriatric patients. Brangan et al.'s (2018) use of NEWS takes chronic low oxygen saturation into account and, if the person is receiving oxygen therapy, the respiratory rate reference values in NEWS are less sensitive compared to MEWS [20].

The non-adjusted reference values challenged the RNs' interpretation of MEWS scores and led to ambiguity in clinical reasoning and decision-making, and they highlighted the possibility of making misjudgements regarding the elderly patients' state of health by solely following MEWS recommendations.

Studies have emphasised that EWS systems are not superior to clinical judgement in detecting critical illness in hospital and prehospital settings $[20,24-28]$. The present study shows that MEWS was used as an additional tool in the clinical reasoning and decision-making process. Furthermore, clinical reasoning and decision-making in geriatric home care patients required comprehensive cognitive and non-cognitive skills $[4,5,14]$.

Both RNs and GPs highlighted the importance of geriatric competence and knowing the patients' physical and psychological function in the habitual state to recognise any changes at an early stage. This specific clinical competence was crucial for the RNs in terms of detecting acute functional decline and for GPs and RNs in collaboration to make the proper clinical decisions for the patient. This ability seems to be closely related to organising for personnel continuity and the proper competences, which, in Norwegian home care, is challenging $[15,16,18]$. However, in the present study, the RNs and GPs expressed that, they knew their patients quite well.

RNs also expressed challenges with compliance related to MEWS trigger recommendations in the context of home care. Studies indicate challenges with the use of EWS systems outside hospital settings and the need for adjustments related to different patient groups and contexts [20,23-25]. These challenges were also identified in this study. RNs stated that trigger recommendations in MEWS were difficult to comply with when working in home care. Unlike in a hospital setting, neither RNs in home care nor GPs see the patient around the clock. In home care, health care personnel see their patients with a wide variety of visit frequencies and they have limited flexibility in work lists and often great geographic distances to the patient and. RNs reasoned and made individual and independent decisions about how follow-up could be handled properly. A score of 1-3 indicated frequent follow-ups or contacting the medical service. RNs highlighted that these scores could be habitual state scores for many patients, and could represent false triggers. False triggers could lead to a culture of non-adherence to follow the trigger recommendations for the escalation of parameters in MEWS. The practise of non-adherence to EWS trigger recommendations is in keeping with a study in a hospital setting, which showed that nurses relied on their own clinical judgement to manage the patient rather than comply with the escalation protocol, and often considered EWS as a task rather than a tool to support clinical decisions [24].

Furthermore, a score of 0 can also be abnormal for a patient with a high score in the habitual state, which could require frequent measures or the need for medical supervision. If MEWS triggered frequent measures, such as every 1-4 hours, RNs reasoned that it was not prudent for the patient to stay at home, and the need for a higher level of care or admission at the Municipal Immediate Assistance Unit or hospital was required. This study shows similar results as a study performed in a community health care setting [20], where MEWS was also used as support in decision-making regarding the escalation of care and whether a higher level of care was needed.

\section{Methodological considerations}

The design of this study implies that a number of criteria have to be addressed to ensure trustworthiness of the reported findings in this paper [37]. We have aimed for transparency in our study. Transparency relates to the explicitness of the method used, and how clearly this is outlined through detailed descriptions of the analytic process [37]. The process is outlined in this paper by giving several examples of coding the interviews, categorisations and main themes (Table 2). We recognize that we as researchers are a part of the process of producing the data and interpreting their meanings. Our pre-understanding and experiences, as healthcare personnel and researchers, influenced the perception and interpretation of the data in this study. However, the rich examples of quotes in this paper, emphasise the aim of transparency and also illustrate our interpretations of the data. 
The study included both RNs and GPs, and sampling was continued until no further information was obtained. Validity was enhanced by assuring variation in age, clinical experience and the representation of two health care professions and representation of all sizes of Norwegian municipalities. A limitation, regarding validity, was that nurses' assistants and unskilled workers were not included in this study. This group could have contributed their experience with identifying acute functional decline, performing and interpreting MEWS and sharing their experiences in collaboration with RNs in home care.

\section{Conclusions}

This study shows that MEWS supported RNs in conducting comprehensive clinical assessments and reasoning when acute functional decline was detected. The interdisciplinary communication and collaboration appeared to be strengthened by using MEWS, and the GP's work was streamlined. However, several limitations were identified regarding the use of MEWS reference values with geriatric patients, which could lead to ambiguity and misjudgements of the patient's state of health. MEWS trigger recommendations were experienced as difficult and inappropriate to use in the context of home care.

This study identified the need for a modified evidence-based EWS tool with reference values and trigger recommendations adjusted for geriatric patients in home care.

\section{Abbreviations}

MEWS: Modified Early Warning Score, TILT: Early Identification of Life-Threatening conditions, GP: General Practitioners, RN: Registered nurses, EWS: Early Warning Score, RSS: Rapid Response Systems, NEWS: National Early Warning Score,

\section{Declarations}

\section{Acknowledgements}

We thank all RNs and GPs in the municipalities of Southern Norway who contributed their time and participated in this study.

\section{Consent for publications}

Not applicable.

\section{Funding}

This study was funded under the Public Sector PhD scheme by The Research Council of Norway (Project number 273141), the municipality of Tvedestrand and the Board of Directors of Eastern Municipalities of Norway. The University of Oslo contributed research time and organisational support.

\section{Availability of data and materials}

Datasets generated and analysed during the current study are not publicly available due to strict ethics regulations in Norway but may be available from the corresponding author upon reasonable request.

\section{Authors' contributions}

$\mathrm{KJ}$ and MK participated in the study conception and design. KJ, MK and LKB participated in interviews. KJ, MK and LKB analysed the data. KJ drafted the manuscript. MK and LKB critically reviewed the analyses and supplied comments on the manuscript. All authors have read and approved the final version of this manuscript. 


\section{Competing interests}

The authors declare that they have no competing interests.

\section{References}

1.The Ministry of health and care services: White paper number 47. The Coordination Reform. Right treatment-at the right placeto the right time. In. Oslo, Norway. 2009.

https://www.regjeringen.no/contentassets/d4f0e16ad32e4bbd8d8ab5c21445a5dc/no/pdfs/stm200820090047000dddpdfs.pdf Accessed 20 oct 2019.

2.The Ministry of health care services: Living all your life - A quality reform for the elderly. In. Oslo, Norway. 2018. https://www.regjeringen.no/contentassets/196f99e63aa14f849c4e4b9b9906a3f8/no/pdfs/stm201720180015000dddpdfs.pdf. Accessed 20 oct 2019.

3. The Norwegian Patient Safety Program, In safe hands 24/7. https://www.pasientsikkerhetsprogrammet.no/omoss/innsatsomr\%C3\%A5der/tidlig-oppdagelse-av-forverret-tilstand. Accessed 16 oct 2019.

4.Bing-Jonsson P, Foss, C, Bjørk, I.T: The competence gap in community care: Imbalance between expected and actual nursing staff competence. Nordic Journal of Nursing Research 2015, 36(1):11.

5.Næss G, Kirkevold, M, Hammer, W, Straand, J, Bruun Wyller, T: Nursing care needs and services utilised by home-dwelling elderly with complex health problems: observational study. BMC Health Services Research 2017, 17(645):10.

6.Vegda K N, J X, Wang L, Tracy C.S, Moineddin R, Upshur R EG: Trends in health services utilization, medication use, and health conditions among older adults: a 2-year retrospective chart review in a primary care practice. BMC Health Services Research 2009, 9(217):7.

7.Hébert R: Functional decline in old age. Canadian Medical Association Journal 1997, 157(8):9.

8.Hsin-Ju Tang H-YJT, Fang-Wen Hu, Ching-Huey Chen: Changes of geriatric syndromes in older adults survived from Intensive Care Unit. Geriatric Nursing 2016, Volume 38(3):219-224.

9.Cigolle C, Langa, KM, Kabeto, MU, Tian, Z, Blaum, C S.: Geriatric conditions and disability: the health and retirement study. (Author abstract)(Clinical report). Annals of Internal Medicine 2007, 147(3).

10.Anpalahan M, Gibson SJ: Geriatric syndromes as predictors of adverse outcomes of hospitalization. Internal Medicine Journal 2008, 38(1):16-23.

11.Bell SP, Vasilevskis EE, Saraf AA, Jacobsen JML, Kripalani S, Mixon AS, Schnelle JF, Simmons SF: Geriatric Syndromes in Hospitalized Older Adults Discharged to Skilled Nursing Facilities. Journal of the American Geriatrics Society 2016, 64(4):715722.

12.Fox M, Persaud M, Maimets I, O'Brien K, Brooks D, Tregunno D, Schraa E,: Effectiveness of Acute Geriatric Unit Care Using Acute Care for Elders Components: A Systematic Review and Meta-Analysis. The American Geriatrics Society 2012, 60(12):9.

13.Buurman B, Hoogerduijn, JG, de Haan, RJ, Abu-Hanna, A, Lagaay, AM, Verhaar, HJ, Schuurmans, MJ, Levi, M, de Rooij, SE: Geriatric Conditions in Acutely Hospitalized Older Patients: Prevalence and One-Year Survival and Functional Decline (Geriatric Conditions: Prevalence and Outcomes). PLOS ONE 2011, 6(11).

14.Higgs J J, MA: Clinical reasoning in the health professions. London: Elsevier Health Sciences.; 2008.

15.The Norwegian Directorate of Health: Professional training in the nursing and care services In.; 2018.

https://www.helsedirektoratet.no/statistikk/statistikk/kvalitetsindikatorer/kommunale-helse-og-omsorgstjenester/fagutdanning-

Page $13 / 16$ 
i-pleie-og-omsorgstjenesten. Accessed 20 Oct 2019.

16.Kattouw CE, Wiig, S: The organisation of community nursing services may impact negatively on safety and the quality of care. Sykepleien Forskning 2019(13):21.

17.The Ministry of health care services: Regulations on quality in the nursing and care services for services provided by the Act of 19 November 1982 No. 66 on health services in the municipalities and by the Act of 13 December 1991 No. 81 on social services, etc. In.;2003. https://lovdata.no/dokument/SF/forskrift/2003-06-27-792. Accessed 20.0ct 2019.

18.Gjevjon ER, Eika, K.H, Romøren, T.I, Landmark, B.F: Measuring interpersonal continuity in high-frequency home healthcare services. Journal of Advanced Nursing 2014, 70(3):11.

19.Dale B, Sævareid, H.I, Kirkevold, M, Sõderhamn, O: Formal and informal care in relation to activities of daily living and selfperceived health among older care-dependent individuals in Norway. International Journal of Older People Nursing 2008, 3(3):10.

20.Brangan E, Banks J, Brant H, Pullybank A, Le Roux H, Redwood, S: Using the National Early Warning Score (NEWS) outside acute hospital settings: a qualitative study of staff experiences in the West of England. BMJ open 2018, 8(10):8.

21.Smith BME, Chiovaro, J.C, O'Neil, M, Kansagara, D, Quiñones, A.R, Freeman, M, Motu'apuaka, M.L, Slatore, C.G: Early Warning System Scores for Clinical Deterioration in Hospitalized Patients: A Systematic Review. AnnalsATS 2014, 11(9):13.

22. Subbe CP, Kruger, M, Rutherford, P, Gemmel, L: Validation of a modified Early Warning Score in medical admissions. Q J Med 2001, 94(10):6.

23.Downey CL, Tahir, W, Randell, R, Brownc, J.M, Jaynea, D.G: Strengths and limitations of early warning scores: A systematic review and narrative synthesis. International Journal of Nursing Studies 2017, 76:14.

24.Foley C, Dowling, M: How do nurses use the early warning score in their practice? A case study from an acute medical unit. Journal of Clinical Nursing 2019, 28(7-8):10.

25.Fox A, Elliot, N: Early warning scores: A sign og deteriation in patients and systems. Nursing management 2015, $22(1): 6$.

26.Jensen JK, Skaar, R, Tveit, B: The impact of Early Warning Score and Rapid Response Systems on nurses' competence: An integrative literature review and synthesis. J Clin Nurs 2017, 27(7-8):19.

27.Fullerton JN, Priceb, C.L, Silveya, N.E, Bracea, S.J, Perkins, G.D: Is the Modified Early Warning Score (MEWS) superior to clinician judgement in detecting critical illness in the pre-hospital environment? Resuscitation 2012, 83(5):6.

28. Williams TA, Tohira, H, Finn, J, Perkins, G.D, Ho, K.M: The ability of early warning scores (EWS) to detect critical illness in the prehospital setting: A systematic review. Recuscitation 2016, 102:8.

29.Patel R, Nugawela, M.D, Edwards, H.B, Richards, A, Le Roux, H, Pullyblank, A, Whiting, P: Can early warning scores identify deteriorating patients in pre-hospital settings? A systematic review. Resuscitation 2018, 132:11.

30.Pedersen L: Early identification of life-threatening conditions In.: Hospital of Sourthern Norway. 2014.

http://ss.prod.fpl.nhn.no/SiteCollectionDocuments/PWA/Pasientsikkertskonferansene/TILT\%20-\%20Line\%20Pedersen.pdf. Accessed 20 Oct 2019.

31.Polit D, Beck, CT: Nursing research: generating and assessing evidence for nursing practice, 9th edn. Philadelphia, Pa: Wolters Kluwer Health; 2012.

32.Justesen L, Mik-Meyer, N: Qualitative research methods in organisation studies. Copengagen: Hans Reitzels Forl.; 2012.

Page $14 / 16$ 
33.Kvale S, Brinkmann, S: InterView[s] learning the craft of qualitative research interviewing 2nd edn. Oslo: Gyldendal akademisk; 2009.

34.Langørgen A LS, Aaberge, R: Grouping of municipalities by population and economic framework conditions 2013,. In. Oslo: Statistics Norway 2013. https://www.ssb.no/offentlig-sektor/artikler-og-publikasjoner/_attachment/225199?_ts = 14ce4c230d0. Accessed 20 Oct 2019.

35.Autotranskription: version 6.2.5 f4transkript In. Edited by Dresing TPuDT. Germany: dr. dresing \& pehl gmbH audiotranskription; 2018. https://www.audiotranskription.de/. Accessed 20 Ocy 2019.

36.QRS International: Nvivo 12 for windows. In.: OSR International; 2018. https://www.qsinternational.com/nvivo/nvivoproducts/nvivo-12-plus. Accessed 20 Oct 2019.

37.Green J, Thorogood, N: Qualitative methods for health research. London: Sage; 2014.

38.Tjora A: Kvalitative forskningsmetoder i praksis [Qualitative research methods in practice], 2nd ed. edn. Oslo: Gyldendal Akademisk; 2012.

\section{Tables}

Table 1: Characteristics of the sample

\begin{tabular}{|l|r|r|r|}
\hline Variable & GP $(\mathbf{n}=8)$ & RN $(\mathbf{n}=35)$ & Totalt $(\mathbf{n}=44)$ \\
\hline Age (mean (SD)) & $43,25(5,94)$ & $43,92(13,26)$ & $43.8(12,2)$ \\
\hline $20-29$ & & & $9(20.5)$ \\
\hline $30-39$ & & & $6(13.6)$ \\
\hline $40-49$ & & & $13(29.5)$ \\
\hline $50-59$ & & & $12(27.3)$ \\
\hline $60-69$ & $15,13(6,33)$ & $15,81(10,89)$ & $15,69(10,16)$ \\
\hline Clinical experience (mean (SD)) & 0 & $6(17,14)$ & $7(15.9)$ \\
\hline $0-5$ & $3(37,5)$ & $6(17,14)$ & $9(20.5)$ \\
\hline $6-10$ & $5(62,5)$ & $11(31,42)$ & $15(34.1)$ \\
\hline $11-20$ & 0 & $8(22,85)$ & $9(20.5)$ \\
\hline $21-30$ & 0 & $4(11,42)$ & $4(9.1)$ \\
\hline $31-40$ & $7(87.5)$ & & $7(87.5)$ \\
\hline Medical specialization, Physicians & $2(25.0)$ & & $2(25.0)$ \\
\hline General medicine & $2(25.0)$ & & $2(25.0)$ \\
\hline Internal medicine & & & \\
\hline Anesthesia & & $4(66.6)$ & $4(66.6)$ \\
\hline Continuing education, Nurses (n=9) & & $1(11.1)$ & $1(11.1)$ \\
\hline Palliative care & & $1(11.1)$ & $1(11.1)$ \\
\hline Dementia care & & $1(11.1)$ & $1(11.1)$ \\
\hline Rehabilitation & & $2(22.2)$ & $2(22.2)$ \\
\hline Geriatric & & &
\end{tabular}

All values are n (\%) unless otherwise specified.

Table 2: Examples of codes, categories, and main themes of the qualitative analysis 
«Many of our care recipients have dementia and it is not in their mind to tell when something is wrong, so you just have to know your care recipients to reason if its normal or not"! (RN-3)

"I think it's something about that continuity and that home care nurses know the care recipients very well, and that is important. It is as important as well as an instrument like MEWS". (GP-6)

"We work a lot with geriatric patients, and I think that MEWS is not adapted to geriatric patients, it's kind of like it should be suitable for me if I'm hospitalized". (RN-4)

"There is a risk of blindly trusting the measurements and not interpreting the clinical situation. [MEWS] is a starting point, but there can be serious misjudgements. " (RN-44)

"I think that what you give of information and the dissemination of the observations that you cannot measure, is as important as these measurements. And it is important that this also is communicated by someone who knows the care recipients and that we can discuss it with the nurses and try to find out what this is all about" (GP-36).

"I think it is easier now [after the introduction of MEWS] to make assessments early in the process. It has changed the course of treatment for the patients. We can initiate medical help early, or we can say no, it's safe to wait. You might avoid unnecessary hospital admissions, for example" (GP-14).

"I see that it matters what weekday it is [when MEWS is used]. If it is Friday and you know in a way that the care recipient [may need medical attention] Saturday or Sunday, then you have to think differently. If it is 14 hours until Monday and the medical office is open again in a way ....there are many things to consider" (RN-20).
Nurses must be in

contact with the

patients to identify

changes

Patients have

parameters outside the

frame of reference in

habitual state.

Care must be taken to

consider the entire

clinical picture, not just

objective

measurements

MEWS can prevent

unnecessary hospital

admissions

Planning and organizing

before the weekend,

holidays and evening
Nurses and

GPs have to

know their

patients

MEWS is not

an

appropriate

tool for all

patient

groups

Competency

in clinical

reasoning

Change of

practice

after the

introduction

of MEWS

Organizing

MEWS as an

additional

clinical decision

making-tool.

the service

\section{Adjusting to the use of MEWS to a non- institutional care setting}

\section{Figures}

\begin{tabular}{|c|c|c|c|c|c|c|c|}
\hline Score & 3 & 2 & 1 & 0 & 1 & 2 & 3 \\
\hline Systolic blood pressure & $<70$ & $71-80$ & $81-100$ & $101-199$ & & $>200$ & \\
\hline Level of consciousness & & & & Alert & Voice & Pain & Unresponsive \\
\hline
\end{tabular}

\begin{tabular}{|c|c|c|}
\hline Color-code & MEWS score & Follow up/new measurements \\
\hline Blue & 0 & 24 hours \\
\hline Yellow & 1 & $8-12$ hours \\
\hline Orange & 2 & $4-8$ hours \\
\hline Red & $3-4$ & $1-4$ hours \\
\cline { 2 - 3 } & $>4$ & Contact physician \\
\hline
\end{tabular}

\section{Figure 1}

